\title{
La democratie en trompe l'oeil \\ RESUMÉ
}

Ce texte discute de l'essence du pouvoir dans une société démocratique et met l'accent sur la question des médias et de la disparition du simbolique.

\section{RESUMO}

Este texto discute a essência do poder numa sociedade democrática e destaca a questão da mídia e do desaparecimento do simbólico.

\section{MOTS-CLÉ (PALAVRAS-CHAVE)}

- Politique (Política)

- Démocratie (Democracia)

- Communication (Comunicação)

\section{Henri-Pierre Jeudy}

\section{CNRS - França}

DANS UN SYSTÈME DÉMOCRATIQUE, les procédures de légitimation du pouvoir sont si permanentes qu'elles paraissent empêcher toute forme d'un totalitarisme naissant. Il n'y a pour ainsi dire aucune possibilité d'exercer un pouvoir absolu. La représentation ostensible de l'autorité passe d'ab-ord par le fonctionnement même des institutions. Cette «raison institutionnelle» représente-t-elle une «individualité collective supérieure» ? Se pose alors la question du maintien de l'individualité dans "l'indi-vidu collectif» que constitue une société. La critique d'Alexis de Tocqueville à l'égard de la démocratie avait essentiellement pour objet le risque d'une uniformisation et d'une disparition de toute forme d'individualité. «Je veux imaginer sous quels traits nouveaux le despotisme pourrait se produire dans le monde : je vois une foule innombrable d'hommes semblables et égaux qui tournent sans repos sur eux-mêmes pour se procurer de petits et vulgaires plaisirs, dont ils emplissent leurs âmes.» ${ }^{1} \mathrm{La}$ «raison institutionnelle», comme expression de cette «individualité collective supérieure», ne serait qu'un principe destructeur du caractère indivis de la souveraineté de l'individu. Alexis de Tocqueville voyait dans la forme tutélaire de l'Etat démocratique l'exercice possible d'un pouvoir absolu et doux, se dispensant de toute violence physique. Cette destruction en douceur de la singularité individuelle s'accomplit au nom d'une nécessité partagée collectivement grâce à l'application rationnelle des règles institutionnelles productrices du sens donné à la vie sociale.

Il n'y aurait pas d'expression possible d'un contre-pouvoir quand l'exercice du pouvoir simule le non-pouvoir dans un jeu de concessions : en démocratie, la négociation perpétuelle est le meilleur moyen de créer cette illusion du partage des pouvoirs. Le seul ennemi désigné d'un système démocratique, c'est l'arbitraire, et pour le combattre, 
il faut bien le voir partout comme une menace. Mais l'arbitraire est aussi la condition de la souveraineté du sujet puisque la revendication de ses droits commence par la dénonciation de l'arbitraire des décisions prises par le pouvoir qui est censé le représenter.

\section{Quelle peuł être l'essence du pouvoir?}

La phrase célèbre de C. Schmitt - «est souverain celui qui décide de la situation exceptionnelle» - laisse bien entendre que, dans un état d'exception ou d'urgence, tout ordre repose sur une décision et non sur le système normatif qui le régit. On pourrait en déduire que la forme absolue de la décision légitime l'exercice d'un pouvoir qui est lui-même absolu. C'est à partir de l'exception que se traduit l'essence de l'autorité de l'Etat, son «unité indivisible». L'acte de décréter un «état d'exception» est une caractéristique du fascisme puisqu'il permet dans le même moment de rendre à la terreur l'apparence de sa nécessité. La dictature fonctionnerait comme un état d'exception constant qui justifierait le pouvoir absolu. Cette percée hors du cadre juridique est de toute évidence le premier danger pour un système démocratique car la situation d'exception n'est pas une justification de l'autonomie de la décision, elle en est la cause directe. L'exercice du pouvoir sort des limites juridiques qui le définissent.

C'est dire que l'essence du pouvoir politique serait définie par occultation délibérée du cadre juridique de son exercice, elle ne pourrait être appréhendée que dans sa forme absolue, hors des limites de la légalité. Selon Hannah Arendt, «la légalité fixe des limites aux activités, mais ne les inspire pas. La grandeur mais aussi l'inconvénient des lois dans les sociétés libres est qu'elles disent uniquement ce que l'on ne devrait pas faire, mais jamais ce que l'on devrait faire.» ${ }^{2}$ La situation d'exception est révélatrice de l'essence du pouvoir puisqu'elle implique au contraire de dire tout ce qu'il faut faire. Si le pouvoir absolu s'installe, son exercice tyrannique impose le mouvement de destruction des libertés. L'épouvantail du totalitarisme permet à toute «société de droit» de légitimer à rebours le «bon fonctionnement» de ses dispositifs normatifs par la fonction d'une représentativité politique qui conjure la menace d'un retour au pouvoir absolu.

Le philosophe Hobbes pensait que le monarque exerçant dans sa propre sphère tous ses caprices évitait à ses concitoyens d'être en proie à l'arbitraire. Il fallait en quelque sorte un lieu clos d'exercice de l'arbitraire pour en résoudre les effets discordants et pour imposer un ordre. L'isolement du monarque aurait été une règle fondamentale de la représentation collective de l'ordre et de la nécessité des règles. Dans son approche anthropologique des sociétés africaines, G.Balandier montre la fonction unificatrice octroyée au «corps souverain» qui, «placé aux frontières de la nature et du social», reste le symbole du pouvoir, rendu visible par «des impositions de comportements et par des rites». «Le souverain est à part, établi dans la singularité, son altérité crée la distance qui permet la domination, l'accès aux forces qui font la société et l'allient au monde.» ${ }^{3}$ La souveraineté, fondée sur une sorte de claustration obligée, devient le principe même du pouvoir et de sa reproduction.

Le pouvoir contiendrait sa propre négation par la clôture même de sa fonction symbolique. Le souverain ne décide plus de rien, à proprement parler, il est là pour représenter la représentation du pouvoir et, dans ce sens, il faut qu'il traduise publiquement, mais de manière implicite, la négation du pouvoir. Ce n'est pas le pouvoir qui pèse sur la tête et sur les épaules du monarque, c'est le port de sa négation comme condition de la représentation de l'ordre et de l'unité. Au Tchad, chez les Moundang, quand le roi est mort, écrit G.Balandier, «ses restes sont ébouillantés afin de pourrir plus vite ; ils sont ensuite enfermés dans 
une urne jetée dans une rivière en crue. Le cadavre royal ne peut retourner à la terre, il la contaminerait et la stériliserait ; il est expulsé, et c'est un mannequin figurant le souverain que le fossoyeur enfouit dans une tombe fictive. Dépouillé de la force du pouvoir, le corps royal n'est plus rien, ou plus justement, il ne porte que le négatif de la royauté». ${ }^{4}$

La mort du roi ou du monarque est aussi un mode de lecture possible de l'essence $d u$ pouvoir. Selon E. Kantorowicz, lors de l'enter-rement des monarques «le cadavre $\mathrm{du}$ roi, son corps naturel, mortel et visible, d'habi-tude - bien qu'ici invisible -, reposait dans une bière de plomb, elle-même enfermée dans un cercueil de bois ; alors que son corps politique, d'habitude invisible, était à cette occasion exposé de façon bien visible par l'effigie revêtue de tous ses insignes royaux officiels : une persona ficta - l'effigie - personnifiant une personna ficta - la Dignitas» ${ }^{5}$ L'hypothèse la plus commune est de penser que les «deux corps» du roi restent unis du vivant de celui-ci, et qu'ils sont séparés le jour de sa mort afin que perdure l'image de sa Majesté. Ce serait là un moyen de conservation du principe même de la souveraineté, la Dignitas du Roi n'étant pas soumise à la contingence. Mais quel serait le pouvoir symbolique de l'effigie sans le cadavre? La séparation des «deux corps du Roi» représente aussi leur unité virtuelle qui est constitutive de l'image publique de la souveraineté. C'est dans la cérémonie de la mort du Roi que son corps politique est rendu visible et qu'en conséquence l'essence du pouvoir royal est présentée aux regards du public (du peuple) par l'effigie. Seulement le cadavre est bien là, dérobé aux mêmes regards, présenté lui aussi comme le signe d'une mortalité qu'il est nécessaire d'oublier. L'effigie sans le «négatif» que représente le cadavre ne pourrait pas assurer son rôle symbolique. Le monarque n'a pas besoin d'être nié pour assurer sa transmission : le «roi ne meurt pas» parce que sa souveraineté contient déjà de son vivant, la négation de son pouvoir par la contingence que son effigie, une fois mort, viendra inverser en figure d'éternité.

En prenant la séparation des deux corps du Roi comme une disjonction dont chaque élément aurait un sens déterminé, on ne tient pas compte de l'implication réciproque des effets de sens entre les deux éléments. Or, il s'agit bien d'un chiasme: de son vivant, le corps du monarque est visible, il montre publiquement sa majesté tandis que son pouvoir demeure invisible, séparé du corps de la société tout en étant le principe de son unité ; lors de sa mort, c'est le pouvoir qui devient visible par la présentation publique de ses attributs symboliques tandis que son corps mort (le cadavre) reste invisible. La souveraineté est fondée sur cette inversion et sur la réciprocité des éléments qui la composent : le pouvoir contient toujours sa négation qui n'apparaît jamais comme telle.

Dans les démocraties contemporaines, la démonstration de l'exercice du pouvoir n'est plus que la confirmation publique d'une gestion transformée en procédure de légitimation morale. Le paradoxe du pouvoir politique en démocratie moderne correspond au fait que l'acquisition irréfutable de la légitimité de l'élu du peuple est en même temps soumise à un mouvement perpétuel de légitimation de ses actes et de ses intentions. La sur-visibilité du pouvoir en action, entretenue par la médiatisation, s'accompagne d'un culte de la transparence qui entraîne la subordination du politique à la règle morale. Au regard des masses, la souveraineté politique devient la figure emblématique du soupçon. Elle laisse trop croire que l'acquisition du pouvoir est un moyen de s'absoudre de toute légitimation de son exercice. La souveraineté n'est-elle alors qu'une métaphore vide pour désigner la forme hégémonique d'un pouvoir?

Comme l'explique bien E. Canetti, le souverain est pris dans une «machine paranoïaque» : le danger est partout, il ne peut le combattre qu'en exerçant indéfiniment son droit de vie et de mort sur ses sujets. Quand il gracie au dernier moment celui 
qu'il a condamné à la mort, il accomplit le geste impérieux de sa suprématie absolue 6 . Le souverain absolu exprime une puissance contre laquelle on ne peut rien et qui n'a pas besoin d'être justifiée. Il ne cesse d'inspirer la crainte. Le principe égalitaire qui est à l'origine de l'idéal démocratique s'oppose de toute évidence à la tyrannie fondée sur un tel assujettissement. L'affirmation de la souveraineté, quand elle n'est pas rendue possible par le suffrage universel, apparaît comme le fruit d'une démesure de l'arbitraire. Et ce fruit ne contient-il pas le germe d'une négation totale des valeurs de la démocratie?

\section{Qu'en est-il de la souveraineté populaire?}

Pour J. Habermas, l'idée de souveraineté populaire demeure pensée dans le cadre d'une réalisation de la démocratie. Si les masses ne participent pas aux prises de décision qui déterminent la métamorphose présente d'une société, l'expression de leur souveraineté reste vide de sens. J. Habermas reconnaît «qu'une souveraineté populaire devenue sans sujets et anonyme, inter-subjectivement dissoute, ne trouve pas exclusivement son expression dans les procédures démocratiques et les exigeantes conditions communicationnelles de son implantation ${ }^{7}$, mais, pour lui, elle n'a de sens que dans la formation de l'opinion et de la volonté collectives. Elle doit agir sur les processus de jugement et de décision en se fondant sur une culture politique. Elle se doit de lutter contre l'auto-légitimation de l'administration, l'espace public fonctionnant comme concept normatif face au risque d'une autonomie institutionnelle du pouvoir politique.

Or la «masse critique», dans la diversité de ses modes d'expression, joue du retournement ou de l'inversion du sens sans être soumise à une quelconque procédure institutionnelle préalable ou finale. Ce qu'on désigne communément comme l'as-pect versatile de l'opinion publique peut fort bien être l'effet d'un jeu de retournement du sens que le pouvoir politique ne maîtrise pas. Et ce jeu est une négation implicite de toute procédure institutionnelle. Mais une pareille hypothèse n'est pas admissible, du point de vue de J. Habermas, si on considère que la souveraineté populaire ne fait sens que dans une perspective d'optimisation de la démocratie.

Entre la «souveraineté populaire» et la «souveraineté des masses», la différence demeure absolue. Deux principes fondamentaux règlent la souveraineté populaire : l'équité partagée par tous et la transcendance du Bien universel. L'équité ne suppose que le juste partage des pouvoirs. La répartition équitable des pouvoirs, fondée sur les principes de légitimité et de reconnaissance publique, semble limiter les risques de dogmatisme, d'hégémonisme, de totalitarisme. Le pouvoir n'a plus vraiment une origine assignable, comparable à celle d'un tyran. Il est partout et nulle part. En fait, il est plutôt partout que nulle part. Mais il n'est plus à combattre puisque, d'une certaine manière, il figure l'expres-sion même de la liberté. Chacun ayant ses chances, le pouvoir est accessible à tous, il suffit de se soumettre aux règles de sa conquête. Cette multiplicité et cette équivalence des formes du pouvoir produisent pourtant des effets destructeurs de toute équité. Ce sont bel et bien les mêmes modèles d'organisation et de distribution du sens qui permettent d'obtenir et d'exercer un certain pouvoir. C'est à partir d'une uniformisation idéologique que la moindre expression d'un pouvoir a des chances de s'affirmer. Le principe égalitaire, qui donne ses chances à chacun, semble préférable à n'importe quel totalitarisme destructeur de l'individualité.

Quand A.Tocqueville adoptait comme critique de la démocratie, le fait que le règne de l'individualisme était fondé sur l'uniformisation, il laissait déjà entendre que «l'esprit d'individualité» était l'ennemi du principe égalitaire : «je vois clairement dans l'égalité deux tendances : l'une qui 
porte l'esprit de chaque homme vers des pensées nouvelles, et l'autre qui le réduirait volontiers à ne plus penser. Et j'aper-çois comment, sous l'empire de certaines lois, la démocratie éteindrait la liberté intellectuelle que l'état social démocratique favorise, de telle sorte qu'après avoir brisé toutes les entraves que lui imposaient jadis des classes ou des hommes, l'esprit humain s'enchaînerait étroitement aux volontés du plus grand nombre. $»^{8}$ Le principe égalitaire, imposé au nom du plus grand nombre fonctionne comme un idéal négatif alors qu'il est présenté comme un idéal positif par ceux qui détiennent le pouvoir et qui démontrent ostensiblement comment il faut appliquer un tel principe. Ils ne sont pas prêts de perdre leur place et encore moins la jouissance qu'elle leur procure. La gestion $\mathrm{du}$ principe égalitaire reste entre leurs mains et ils ne manquent pas de l'utiliser à leurs propres fins. Il ne s'agit pas d'une simple supercherie qui consisterait à entretenir les illusions des autres. L'égalité des chances est paradoxalement invoquée pour démontrer qu'il n'y a pas de place pour tout le monde. Le principe égalitaire est une machine à détruire l'expres-sion de la souveraineté des masses par le nivellement du pouvoir.

L'autre principe est celui de la reconnaissance toujours partagée d'un bien commun, ou de la fonction transcendantale du bien. Dès qu'il s'agit de la souveraineté du Bien, le fait même de sa transcendance et de sa fonction morale entraîne un consensus. Le sujet abandonne ainsi sa propre souveraineté, qui a partie liée avec la tentation $\mathrm{du}$ Mal, pour se rallier à celle du Bien qui, par sa perspective atemporelle et universelle, demeure une garantie idéale pour le devenir de l'humanité. L'hypothèse inverse, celle qui consiste à croire que le Mal est l'avenir de l'homme est réduit à son expression nihiliste puisqu'elle laisse supposer que l'humanité œuvre à sa propre destruction d'une manière inéluctable. Comme la reconnaissance de la souveraineté du Bien est le refuge idéal contre l'arbitraire du sujet, c'est donc au prix d'une capitulation acceptée par le sujet lui-même que s'impose le règne du souverain Bien. Quand Iris Murdoch ${ }^{9}$ insiste sur le caractère indéfinissable du concept de Bien et simultanément sur son pouvoir unificateur, elle montre en quoi ce «concept des concepts» est partout présent, tout en dénonçant les contrefaçons qu'il ne cesse de subir. Mais la masse autant que le sujet restent sceptiques à l'égard de cette volonté du Bien dont ils subissent les effets pervers que les media mettent en scène.

\section{Les media ef la disparition du symbolique}

Même si les ethnologues s'acharnent à démontrer combien le pouvoir politique a besoin pour être exercé d'une mise en scène, la dimension de son rôle symbolique semble bien avoir disparu. Les simagrées des leaders ne conjurent pas l'absence de tout échange symbolique. Et la représentativité qui est le fleuron des démocraties use des artifices d'un décorum symbolique devenu lui-même archéologique. Pour tout dire : le symbolique est à la charge exclusive de la conservation patrimoniale! Le jeu de la participation démocratique, telle une concaténation de compromis, masquant mal le vide politique. Le pouvoir politique survit de la mécanique ostensible de sa reproduction parce qu'il ne peut échapper à son destin d'être accompli. Dépourvu de sa puissance symbolique, il est devenu l'exercice d'une gestion dont l'apparence d'optimisation a besoin de la mise en scène médiatique d'une idéologie victimaire universelle. Le processus de revendication de «droits à » impose un sens unique à toute manifestation collective dont la relation au pouvoir passe nécessairement par la reconnaissance préalable du statut de victime. Ainsi en estil de cette cohorte des «droits à » qui signe l'effondrement de la souveraineté de toute expression collective publique.

Se mettant hors jeu par rapport à cette 
idéologie victimaire, le terrorisme qui permet de justifier un état d'exception, et par voie de conséquence, des formes totalitaires du pouvoir, réintroduit dans le monde une dimension symbolique à laquelle le système démocratique et libéral ne semble pas pouvoir répondre. Les Islamistes intégristes, dans leur surenchère à la déstructuration de l'ordre mondial, jouent la logique symbolique que les Occidentaux ont évacuée pour réussir à imposer leur système libéral dans lequel les symboles ne sont plus que des signes pétrifiés et commémorés. Exacerbant la violence primitive des passions, les Islamistes radicaux stigmatisent l'artifice mnémonique du symbolique dans la perpétuation d'un impossible échange.

En développant l'uniformisation universelle de la communication, ce sont les media qui ont anéanti la puissance de l'échange symbolique. Les critiques faites à l'égard de leur fonctionnement, la revendication d'une qualité supérieure des programmes de la télévision ne changent en rien leur capacité à modeler les représentations mentales collectives. Ayant trouvé une fin en elle-même, la communication médiatique s'exerce comme un pouvoir formel absolu, apte à absorber ce qui la nie sans jamais courir le risque de faire émerger ses propres contradictions. L'évidence de son fonctionnement totalitaire n'appa-raît pas parce qu'elle demeure occultée par la certitude de la liberté d'information. Le principe est simple : si tout peut être montré et dit, alors le totalitarisme de la médiatisation n'est qu'une calomnie. La preuve donnée est aussi simple: sans les media, bien des injustices graves, bien des crimes crapuleux ne seraient pas dévoilés à l'opinion publique internationale. Seulement, les média eux-mêmes, se soutenant du rythme infernal de leur exhibitionnisme, anéantissent toute relation symbolique en lui substituant leur unique pouvoir de fascination par exclusion de l'échange.

Cette violence irréductible des media n'apparaît pas comme telle, elle demeure occultée par la convention universelle de leur utilité publique. Le pouvoir politique ne sera plus jamais en mesure de manifester une quelconque singularité qui le caractériserait parce qu'il est acculé à soutenir médiatiquement sa propre légitimité. Son hypothétique souveraineté est soumise au rythme des procédures juridiques qui servent de garantie publique à son apparente intégrité morale. On comprend pourquoi personne ne voudra plus du pouvoir. La seule possibilité d'en jouir est de détenir le contrôle des media pour éviter d'en être la victime.

L'exhibitionnisme universel des media fait du réel, de ce qui advient dans le monde, une image qui n'a plus besoin d'être réfléchie, qui se dispense du processus de la représentation. La médiatisation conjugue les habitudes mentales trans-culturelles à un déroulement perpétuel des images qui signifient ce qu'est la réalité dans un moment toujours rendu atemporel. Ce rythme d'une réflexivité sans fin qui abolit la singularité des points de vue provoque la mort de toute communauté par la mondialisation d'un « être ensemble »

\section{Notes}

1 Alexis TOCQUEVILLE, De la démocratie en Amérique, Tome 2, Librairie Charles Gosselin, Paris, p. 265

2 Hannah ARENDT, Le système totalitaire, p. 213, Points-Seuil, Paris, 1973.

3 Georges BALANDIER, Le Détour, p. 34, Fayard, Paris 1985.

4 G. BALANDIER, texte déjà cité, p. 39

5 Ernst KANTOROWICZ, Les deux corps du Roi, p. 303, Gallimard, Paris, 1989.

6 Elias CANETTI, Masse et Puissance, p. 246, Gallimard, Paris, 1966

7 Jürgen HABERMAS, "La souveraineté populaire comme procédure", Revue Lignes n 7 , p. 52, Librairie Séguier, Paris, 1989 
8 Alexis TOCQUEVILLE, De la démocratie en Amérique, tome II, Edition Nolla, 1990, p.25

9 Iris MURDOCH, La souveraineté du bien, Editions de l'Eclat, Paris, 1994. 